Department of Mathematics

The Relation Between Primal and Dual Boundary Conditions for Hyperbolic Systems of Equations

Fatemeh Ghasemi and Jan Nordström 
Linköping University

Department of Mathematics

SE-581 83 Linköping 


\title{
The Relation Between Primal and Dual Boundary Conditions for Hyperbolic Systems of Equations
}

\author{
Fatemeh Ghasemi \& Jan Nordström \\ Computational Mathematics, Department of Mathematics, Linköping University, \\ SE-581 83 Linköping, Sweden \\ fatemeh.ghasemi@liu.se, jan.nordstrom@liu.se
}

\begin{abstract}
In this paper we study boundary conditions for linear hyperbolic systems of equations and the corresponding dual problems. In particular, we show that the primal and dual boundary conditions are related by a simple scaling relation. It is also shown that the weak dual problem can be derived directly from the weak primal problem.

Based on the continuous analysis, we discretize and perform computations with a high-order finite difference scheme on summation- by-parts form with weak boundary conditions. It is shown that the results obtained in the continuous analysis lead directly to stability results for the primal and dual discrete problems. Numerical experiments corroborate the theoretical results.

Keywords: Hyperbolic systems, boundary conditions, primal problem, dual problem, well-posedness, dual consistency
\end{abstract}

\section{Introduction}

In many engineering applications, functionals computed from the solution, such as for example the lift and drag coefficients in fluid dynamics, are more interesting than the solution of the equations. The importance of duality in the computation of functionals has been investigated in $[1,2,3]$.

More, recently it has been shown that discretizing the equations in a dual consistent way, increase the order of accuracy of the output functional $[1,4,5,6,7]$. This superconvergent behavior has also been investigated for schemes based on Summation-by-Parts (SBP) form in combination with the Simultaneous Approximation Term (SAT) technique in $[8,9,10,11,12]$. 
The only requirement for having a dual consistent scheme on SBP-SAT form is choosing a specific subset of coefficients in the SATs for which stability is guaranteed. Superconvergence of linear integral functionals hence comes with no additional computational costs.

In this article, we study linear hyperbolic systems of equations and focus on the relation between the primal and dual boundary conditions. We also show that the weak dual problem can be derived directly from the weak primal problem in a dual consistent manner.

The remainder of this article proceeds as follows. In Section 2, we define the continuous primal problem, derive the dual (or adjoint) problem and investigate the relation between primal and dual boundary conditions. The requirements for well-posedness on the boundary operators also discussed in this section. Section 3 presents a weak implementation of continuous primal and dual problems. In Section 4, the semi-discrete version of the primal and dual equations and the SAT terms for the boundary conditions are derived, and stability and dual consistency are discussed. Related numerical experiments are performed in Section 5.

\section{The continuous problem}

\subsection{The primal problem}

Consider the following constant coefficient hyperbolic system of equations on the finite domain $\Omega$

$$
\begin{aligned}
& u_{t}+A u_{x}+B u_{y}=F(x, y), \quad(x, y) \in \Omega, \quad t \geq 0 \\
& L_{p} u=0, \quad(x, y) \in \partial \Omega, \quad t \geq 0 \\
& u=0, \quad(x, y) \in \Omega, \quad t=0
\end{aligned}
$$

including homogeneous boundary and initial conditions. In (1), $A$ and $B$ are symmetric constant matrices of size $m \times m$ and $L_{p}$ is the boundary operator acting on the boundary $\partial \Omega$. The data in the problem is the forcing function $F$. If $A, B$ are not symmetric, we start by symmetrizing them [13].

By applying the energy method to (1) (for $F=0$ ) we find

$$
\frac{d}{d t}\|u\|^{2}=-\oint_{\partial \Omega} u^{T} C u d s, \quad C=n_{x} A+n_{y} B, \quad(x, y) \in \partial \Omega,
$$

where $\|u\|^{2}=\oint_{\partial \Omega} u^{T} u d \Omega$ and $n=\left(n_{x}, n_{y}\right)$ is the outward pointing unit normal vector. Note that $C$ varies along $\partial \Omega$, but that it is always symmetric. 
Due to the fact that $C$ is symmetric, we have

$$
C=X \Lambda X^{T}, \quad X=\left[X_{+}, X_{-}\right], \quad \Lambda=\operatorname{diag}\left(\Lambda^{+}, \Lambda^{-}\right),
$$

where $\Lambda$ and $X$ contain the positive and negative eigenvalues and the corresponding orthogonal eigenvectors, respectively. For ease of presentation, and with no restriction, we have assumed that there are no zero eigenvalues. To bound the energy of the solution, boundary conditions must be chosen such that

$$
u^{T} C u=W^{T} \Lambda W=\left(W^{+}\right)^{T} \Lambda^{+} W^{+}+\left(W^{-}\right)^{T} \Lambda^{-} W^{-} \geq 0, \quad(x, y) \in \partial \Omega,
$$

where $W=X^{T} u, W^{+}=X_{+}^{T} u$ and $W^{-}=X_{-}^{T} u$.

\subsection{The dual problem}

Consider the linear functional

$$
J(u, G)=\int_{\Omega} u^{T} G d \Omega,
$$

where $G$ is a vector weight function. To derive the dual problem, we search for the dual solution $\phi$ such that $J(u, G)=J(\phi, F)$. By using integration by parts, we find

$$
\begin{aligned}
\int_{0}^{T} J(u, G) d t & =\int_{0}^{T} J(u, G) d t-\int_{0}^{T}\left(\phi, u_{t}+A u_{x}+B u_{y}-F\right) d t \\
& =\int_{0}^{T} J(\phi, F) d t-\left.(\phi, u)\right|_{0} ^{T}-\int_{0}^{T} \oint_{\partial \Omega} \phi^{T} C u d s d t \\
& +\int_{0}^{T}\left(\phi_{t}+A \phi_{x}+B \phi_{y}+G, u\right) d t .
\end{aligned}
$$

It follows that the dual end condition is $\phi(x, y, T)=0$. Furthermore, the dual boundary conditions $L_{d} \phi=0$ must be such that

$$
\phi^{T} C u=\Psi^{T} \Lambda W=\left(\Psi^{+}\right)^{T} \Lambda^{+} W^{+}+\left(\Psi^{-}\right)^{T} \Lambda^{-} W^{-}=0, \quad(x, y) \in \partial \Omega,
$$

when the primal conditions are applied. In (4), $\Psi=X^{T} \phi, \Psi^{+}=X_{+}^{T} \phi$ and $\Psi^{-}=X_{-}^{T} \phi$. Finally, the dual equation becomes

$$
-\phi_{t}-A \phi_{x}-B \phi_{y}=G, \quad(x, y) \in \Omega .
$$


By introducing the time transformation $\tau=T-t$, the dual problem becomes

$$
\begin{array}{rlrlrl}
\phi_{\tau}-A \phi_{x}-B \phi_{y} & =G, & & (x, y) \in \Omega, & & \tau \geq 0 \\
L_{d} \phi & =0, & & (x, y) \in \partial \Omega, & \tau \geq 0 \\
\phi & =0, & & (x, y) \in \Omega, & & \tau=0 .
\end{array}
$$

The energy method applied to (5) with $G=0$ results in

$$
\frac{d}{d \tau}\|\phi\|^{2}=+\oint_{\partial \Omega} \phi^{T} C \phi d s
$$

which implies that an energy estimate is obtained if the dual boundary conditions are chosen such that

$$
\phi^{T} C \phi=\Psi^{T} \Lambda \Psi=\left(\Psi^{+}\right)^{T} \Lambda^{+} \Psi^{+}+\left(\Psi^{-}\right)^{T} \Lambda^{-} \Psi^{-} \leq 0, \quad(x, y) \in \partial \Omega .
$$

\subsection{The relation between primal and dual boundary conditions}

In this section, the relation between the primal and dual boundary conditions is investigated where we assume that both lead to well-posed problems. The conditions (3) and (7) will be discussed in detail later. The general form of boundary conditions for the primal and dual problems are

$$
W^{-}=R_{1} W^{+}, \quad \Psi^{+}=S_{1} \Psi^{-},
$$

respectively. Next, we investigate the relation between $R_{1}$ and $S_{1}$ such that (4) holds.

In the boundary conditions (8), we specify the ingoing characteristic variables in terms of the outgoing ones, which is necessary for well-posedness as can be seen from (2),(3) and (6),(7). The number of rows in matrices $R_{1}$ and $S_{1}$ are equal to the number of boundary conditions for the primal and dual problem, respectively $[14,15]$.

The relation (4) can now be rewritten as

$$
\phi^{T} C u=\Psi^{T} \Lambda W=\tilde{\Psi}^{T} \tilde{\mathcal{S}}^{T} \Lambda \tilde{\mathcal{R}} \tilde{W}, \quad \tilde{\mathcal{R}}=\left[\begin{array}{cc}
I & 0 \\
R_{2} & I
\end{array}\right], \quad \tilde{\mathcal{S}}=\left[\begin{array}{cc}
I & S_{2} \\
0 & I
\end{array}\right]
$$

where $\tilde{W}=\tilde{\mathcal{R}}^{-1} W=\left[W^{+}, W^{-}-R_{2} W^{+}\right]^{T}, \tilde{\Psi}=\tilde{\mathcal{S}}^{-1} \Psi=\left[\Psi^{+}-S_{2} \Psi^{-}, \Psi^{-}\right]^{T}$ and

$$
\tilde{\mathcal{S}}^{T} \Lambda \tilde{\mathcal{R}}=\left[\begin{array}{cc}
\Lambda^{+} & 0 \\
S_{2}^{T} \Lambda^{+}+\Lambda^{-} R_{2} & \Lambda^{-}
\end{array}\right]
$$


Now demanding that (8) holds, i.e. that $R_{2}=R_{1}$, and $S_{2}=S_{1}$ leads to

$$
\phi^{T} C u=\left(\Psi^{-}\right)^{T}\left(S_{1}^{T} \Lambda^{+}+\Lambda^{-} R_{1}\right) W^{+},
$$

which vanish if

$$
S_{1}=-\left(\Lambda^{+}\right)^{-1} R_{1}^{T} \Lambda^{-} \quad \text { or equivalently } \quad R_{1}=-\left(\Lambda^{-}\right)^{-1} S_{1}^{T} \Lambda^{+} .
$$

We have proven

Proposition 1. The relation between the primal and dual boundary conditions for the problem (1) and (5) is given by (8) and (9).

Remark 1. This implies that as soon as the primal/dual boundary conditions are given, the corresponding dual/primal boundary conditions are obtained by a simple scaling operation.

In order to obtain symmetric formulations for both the primal and dual boundary conditions, a rescaled version of $R_{1}$

$$
R_{1}=+\left(\Lambda^{-}\right)^{-1} R,
$$

will be used in the rest of the paper. The matrix $R$ has the same size as $R_{1}$. By inserting (10) into (9), we get

$$
S_{1}=-\left(\Lambda^{+}\right)^{-1} R^{T} .
$$

The relations (10) and (11) implies that the general form of primal and dual boundary conditions given in (8) can now be written as

$$
\begin{aligned}
& L_{p} u=\left(\Lambda^{-} X_{-}^{T}-R X_{+}^{T}\right) u=\Lambda^{-} W^{-}-R W^{+}=0, \\
& L_{d} \phi=\left(\Lambda^{+} X_{+}^{T}+R^{T} X_{-}^{T}\right) \phi=\Lambda^{+} \Psi^{+}+R^{T} \Psi^{-}=0 .
\end{aligned}
$$

\subsection{Well-posedness of primal problem}

The energy rate given in (2) including the boundary conditions (12) now becomes

$$
\frac{d}{d t}\|u\|^{2}=-\oint_{\partial \Omega}\left(W^{+}\right)^{T}\left(\Lambda^{+}+R^{T}\left(\Lambda^{-}\right)^{-1} R\right) W^{+} d s .
$$

In order to obtain an energy estimate, the matrix $R$ must satisfy

$$
\Lambda^{+}+R^{T}\left(\Lambda^{-}\right)^{-1} R \geq 0 .
$$


Remark 2. $R=0$ yields the so called characteristic boundary conditions.

Since we have used a minimal number of boundary conditions necessary for existence, and the energy estimate leads to uniqueness, we can state

Proposition 2. The primal problem (1) with the boundary conditions (12), subject to condition (13) is well-posed.

\subsection{Well-posedness of dual problem}

Following the procedure for the primal problem, we rewrite (6) with the dual boundary conditions (12) as

$$
\frac{d}{d \tau}\|\phi\|^{2}=+\oint_{\partial \Omega}\left(\Psi^{-}\right)^{T}\left(\Lambda^{-}+R\left(\Lambda^{+}\right)^{-1} R^{T}\right) \Psi^{-} d s .
$$

To obtain an energy estimate, the matrix $R$ (in addition to (13)) must satisfy

$$
\Lambda^{-}+R\left(\Lambda^{+}\right)^{-1} R^{T} \leq 0 .
$$

Remark 3. $R=0$ yields the characteristic boundary conditions for (5).

The result is summarized in the following proposition

Proposition 3. The dual problem (5) with the boundary conditions (12), subject to condition (14) is well-posed.

We can also prove

Proposition 4. The dual boundary conditions given in (12) leads to an energy-estimate of dual problem if and only if the primal boundary conditions in (12) leads to an energy-estimate for the primal problem.

Proof. Consider the symmetric matrix

$$
N=\left[\begin{array}{cc}
\Lambda^{+} & R^{T} \\
R & -\Lambda^{-}
\end{array}\right]
$$

If the primal problem has an energy-estimate, then (13) holds. This implies that the Schur complement $\Lambda^{+}+R^{T}\left(\Lambda^{-}\right)^{-1} R$ of $N$ is positive semi-definite, which leads to $N \geq 0$. Since $N \geq 0$ and $\Lambda^{+}>0$, the other Schur complement $-\left(\Lambda^{-}+R\left(\Lambda^{+}\right)^{-1} R^{T}\right)$ is also positive semi-definite and therefore the dual problem has an energy-estimate, i.e. (14) holds. Conversely, if the dual problem has an energy-estimate, then (14) holds which leads to $N \geq 0$. Since $N \geq 0$ and $-\Lambda^{-}>0$, the Schur complement $\Lambda^{+}+R^{T}\left(\Lambda^{-}\right)^{-1} R$ is positive semi-definite and hence the primal problem has an energy-estimate, i.e. (13) holds. 
Remark 4. We summarize the results so far:

- The primal problem is well-posed if (3) holds.

- The dual problem is well-defined if (4) holds.

- The dual problem is well-posed if (7) holds.

- Primal/dual well-posed boundary conditions yield dual/primal well-posed boundary conditions by a simple scaling operation.

\section{Weakly imposed boundary conditions}

\subsection{Weakly imposed primal boundary conditions}

The boundary conditions (12) can also be imposed by adding a penalty term to the right-hand side of (1). This can be formulated as

$$
\begin{aligned}
& u_{t}+A u_{x}+B u_{y}=\mathcal{L}\left(\Sigma_{p}\left(L_{p} u\right)\right)+F, \quad(x, y) \in \Omega, \quad t \geq 0 \\
& u=0, \quad(x, y) \in \Omega, \quad t=0
\end{aligned}
$$

where $\mathcal{L}$ is a lifting operator [16] defined by $\int_{\Omega} \phi^{T} \mathcal{L}(\psi) d \Omega=\oint_{\partial \Omega} \phi^{T} \psi d s$ for smooth vector functions $\phi, \psi$. In (15), $\Sigma_{p}$ is a penalty matrix yet to be determined.

The energy method applied to (15) with $F=0$ leads to

$$
\begin{aligned}
\frac{d}{d t}\|u\|^{2}= & -\oint_{\partial \Omega}\left(\left(W^{+}\right)^{T} \Lambda^{+} W^{+}+\left(W^{-}\right)^{T} \Lambda^{-} W^{-}\right) d s \\
& +\oint_{\partial \Omega} u^{T} \Sigma_{p}\left(\Lambda^{-} W^{-}-R W^{+}\right)+\left(u^{T} \Sigma_{p}\left(\Lambda^{-} W^{-}-R W^{+}\right)\right)^{T} d s .
\end{aligned}
$$

Choosing

$$
\Sigma_{p}=\alpha X_{-}
$$

as the penalty matrix with a yet unknown scalar parameter $\alpha$ yields

$$
\frac{d}{d t}\|u\|^{2}=-\oint_{\partial \Omega}\left[\begin{array}{l}
W^{+} \\
W^{-}
\end{array}\right]^{T} \underbrace{\left[\begin{array}{cc}
\Lambda^{+} & \alpha R^{T} \\
\alpha R & (1-2 \alpha) \Lambda^{-}
\end{array}\right]}_{M}\left[\begin{array}{l}
W^{+} \\
W^{-}
\end{array}\right] d s .
$$

The matrix $M=M(\alpha)$ in (17) can be rotated into block diagonal form

$$
M^{\text {rot }}=\mathcal{T}^{T} M \mathcal{T}, \quad \mathcal{T}=\left[\begin{array}{cc}
I & 0 \\
\mathcal{T}_{21} & I
\end{array}\right], \quad \mathcal{T}_{21}=-\frac{\alpha}{1-2 \alpha}\left(\Lambda^{-}\right)^{-1} R,
$$


leading to

$$
M^{r o t}=\left[\begin{array}{cc}
\Lambda^{+}-\frac{\alpha^{2}}{1-2 \alpha} R^{T}\left(\Lambda^{-}\right)^{-1} R & 0 \\
0 & (1-2 \alpha) \Lambda^{-}
\end{array}\right] .
$$

The matrix $M^{\text {rot }}$ is positive semi-definite if $\alpha$ is chosen such that

$$
\alpha>\frac{1}{2}, \quad \Lambda^{+}-\frac{\alpha^{2}}{1-2 \alpha} R^{T}\left(\Lambda^{-}\right)^{-1} R \geq 0
$$

holds. The matrix $\mathcal{T}_{21}$ has linearly independent rows and we find that the new variables are $\mathcal{T}^{-1} W=\left[W^{+}, W^{-}-\mathcal{T}_{21} W^{+}\right]^{T}$.

We summarize the result in

Proposition 5. The primal problem (1) with the boundary conditions (12) implemented weakly as in (15),(16) leads to well-posedness if (18) holds.

\subsection{Weakly imposed dual boundary conditions}

By imposing the dual boundary conditions (12) weakly, we have

$$
\begin{aligned}
& \phi_{\tau}-A \phi_{x}-B \phi_{y}=\mathcal{L}\left(\Sigma_{d}\left(L_{d} \phi\right)\right)+G, \quad(x, y) \in \Omega, \quad \tau \geq 0 \\
& \phi=0, \quad(x, y) \in \Omega, \quad \tau=0 .
\end{aligned}
$$

The result of the energy method applied to (19) (letting $G=0$ ) yields

$$
\begin{aligned}
\frac{d}{d \tau}\|\phi\|^{2}= & +\oint_{\partial \Omega}\left(\left(\Psi^{+}\right)^{T} \Lambda^{+} \Psi^{+}+\left(\Psi^{-}\right)^{T} \Lambda^{-} \Psi^{-}\right) d s \\
& +\oint_{\partial \Omega} \phi^{T} \Sigma_{d}\left(\Lambda^{+} \Psi^{+}+R^{T} \Psi^{-}\right)+\left(\phi^{T} \Sigma_{d}\left(\Lambda^{+} \Psi^{+}+R^{T} \Psi^{-}\right)\right)^{T} d s .
\end{aligned}
$$

By choosing

$$
\Sigma_{d}=-\beta X_{+}
$$

where the scalar parameter $\beta$ will be determined later, we obtain

$$
\frac{d}{d \tau}\|\phi\|^{2}=-\oint_{\partial \Omega}\left[\begin{array}{l}
\Psi^{+} \\
\Psi^{-}
\end{array}\right]^{T} \underbrace{\left[\begin{array}{cc}
-(1-2 \beta) \Lambda^{+} & \beta R^{T} \\
\beta R & -\Lambda^{-}
\end{array}\right]}_{\tilde{M}}\left[\begin{array}{l}
\Psi^{+} \\
\Psi^{-}
\end{array}\right] d s .
$$

The matrix $\tilde{M}=\tilde{M}(\beta)$ can be diagonalized as

$$
\tilde{M}^{r o t}=\tilde{\mathcal{T}}^{T} M \tilde{\mathcal{T}}, \quad \tilde{\mathcal{T}}=\left[\begin{array}{cc}
I & \tilde{\mathcal{T}}_{12} \\
0 & I
\end{array}\right], \quad \tilde{\mathcal{T}}_{12}=\frac{\beta}{1-2 \beta}\left(\Lambda^{+}\right)^{-1} R^{T},
$$


leading to

$$
\tilde{M}^{r o t}=\left[\begin{array}{cc}
-(1-2 \beta) \Lambda^{+} & 0 \\
0 & -\Lambda^{-}+\frac{\beta^{2}}{1-2 \beta} R\left(\Lambda^{+}\right)^{-1} R^{T}
\end{array}\right] .
$$

The matrix $\tilde{M}^{\text {rot }}$ is positive semi-definite if $\beta$ is chosen such that

$$
\beta>\frac{1}{2}, \quad-\Lambda^{-}+\frac{\beta^{2}}{1-2 \beta} R\left(\Lambda^{+}\right)^{-1} R^{T} \geq 0
$$

holds. The matrix $\tilde{\mathcal{T}}_{12}$ has linearly independent rows and the new variables are $\tilde{\mathcal{T}}^{-1} \Psi=\left[\Psi^{-}-\tilde{\mathcal{T}}_{12} \Psi^{+}, \Psi^{-}\right]^{T}$.

We summarize the result in

Proposition 6. The dual problem (5) with the boundary conditions (12) implemented weakly as in (19),(20) leads to well-posedness if (22) holds.

Remark 5. The matrix $M(1)$ in (17) is equal to the matrix $\tilde{M}(1)$ in (21). This suggests that the weak primal problem (15) is well-posed if the weak dual problem (19) is well-posed and vice versa. We will return to this issue later.

3.3. A direct path from the weak primal problem to the weak dual problem

We will next show that one can proceed directly from the weak primal problem to the weak dual problem, and at the same time determine the parameters $\alpha, \beta$ in the penalty matrices above. The weak dual boundary formulation will be obtained as a natural requirement for the final formulation.

The well-posed weak primal problem is

$$
\begin{aligned}
u_{t}+A u_{x}+B u_{y} & =\mathcal{L}\left(\alpha X_{-}\left(L_{p} u\right)\right)+F, & & (x, y) \in \Omega, \quad t \geq 0 \\
u & =0, & & (x, y) \in \Omega, \quad t=0 .
\end{aligned}
$$

In the usual manner, we seek the dual solution $\phi$ such that $\int_{0}^{T} J(u, G)=$ $\int_{0}^{T} J(\phi, F)$. Integration by parts gives

$$
\begin{aligned}
\int_{0}^{T} J(u, G) d t & =\int_{0}^{T} J(u, G) d t-\int_{0}^{T}\left(\phi, u_{t}+A u_{x}+B u_{y}-\mathcal{L}\left(\alpha X_{-}\left(L_{p} u\right)\right)-F\right) d t \\
& =\int_{0}^{T} J(\phi, F) d t-\left.(\phi, u)\right|_{0} ^{T}+\int_{0}^{T}\left(\phi_{t}+A \phi_{x}+B \phi_{y}+G, u\right) d t \\
& -\int_{0}^{T} \oint_{\partial \Omega} \phi^{T} C u d s d t+\alpha \int_{0}^{T} \oint_{\partial \Omega} \phi^{T} X_{-} L_{p} u d s d t .
\end{aligned}
$$


The boundary terms on the last row in (24) can be rewritten as

$$
\begin{aligned}
-\oint_{\partial \Omega} \phi^{T}\left(C-\alpha X_{-} L_{p}\right) u d s=-\oint_{\partial \Omega} u^{T}\left(C^{T}-\alpha L_{p}^{T} X_{-}^{T}\right) \phi d s \\
=-\int_{\Omega} u^{T} \mathcal{L}\left[\left(C-\alpha L_{p}^{T} X_{-}^{T}\right) \phi\right] d \Omega \\
=-\int_{\Omega}\left(\mathcal{L}\left[\left(C-\alpha L_{p}^{T} X_{-}^{T}\right) \phi\right]\right)^{T} u d \Omega=\left(\mathcal{L}\left[\left(C-\alpha L_{p}^{T} X_{-}^{T}\right) \phi\right], u\right) \\
=-\left(\mathcal{L}\left[X_{+}\left(\Lambda^{+} \Psi^{+}+\alpha R^{T} \Psi^{-}\right)-(\alpha-1) X_{-} \Lambda^{-} \Psi^{-}\right], u\right) .
\end{aligned}
$$

Inserting the initial and end conditions $u(x, y, 0)=\phi(x, y, T)=0$ and substituting (25) into (24) leads to

$$
\begin{aligned}
\int_{0}^{T} J(u, G) d t & =\int_{0}^{T} J(\phi, F) d t+\int_{0}^{T}\left(\phi_{t}+A \phi_{x}+B \phi_{y}+G, u\right) d t \\
& -\int_{0}^{T}\left(\mathcal{L}\left[X_{+}\left(\Lambda^{+} \Psi^{+}+\alpha R^{T} \Psi^{-}\right)-(\alpha-1) X_{-} \Lambda^{-} \Psi^{-}\right], u\right) d t .
\end{aligned}
$$

The result (26) should of course be identical to the dual formulation (19). By adding and subtracting the dual boundary term $\beta X_{+}\left(L_{d} \phi\right)$ in (19) to (26), we get

$$
\begin{aligned}
& \int_{0}^{T} J(u, G) d t=\int_{0}^{T} J(\phi, F) d t+\int_{0}^{T}\left(\phi_{t}+A \phi_{x}+B \phi_{y}+G, u\right) d t \\
- & \int_{0}^{T}\left(\mathcal{L}\left[\beta X_{+}\left(\Lambda^{+} \Psi^{+}+R^{T} \Psi^{-}\right)\right], u\right) d t \\
- & \int_{0}^{T}\left(\mathcal{L}\left[X_{+}\left((1-\beta) \Lambda^{+} \Psi^{+}+(\alpha-\beta) R^{T} \Psi^{-}\right)-(\alpha-1) X_{-} \Lambda^{-} \Psi^{-}\right], u\right) d t .
\end{aligned}
$$

The specific choices $\alpha=\beta=1$ removes the last term in (27) and yields

$$
\int_{0}^{T} J(u, G) d t=\int_{0}^{T} J(\phi, F) d t+\int_{0}^{T}\left(\phi_{t}+A \phi_{x}+B \phi_{y}+G-\mathcal{L}\left(X_{+} L_{d} \phi\right), u\right) d t .
$$

The last term in (28) cancels if

$$
-\phi_{t}-A \phi_{x}-B \phi_{y}=G-\mathcal{L}\left(X_{+}\left(L_{d} \phi\right)\right) .
$$


Finally, the time-transformation $\tau=T-t$ yields the dual problem

$$
\begin{aligned}
& \phi_{\tau}-A \phi_{x}-B \phi_{y}=G-\mathcal{L}\left(X_{+}\left(L_{d} \phi\right)\right) \quad(x, y) \in \Omega, \quad \tau \geq 0 \\
& \phi=0 \quad(x, y) \in \Omega, \quad \tau=0
\end{aligned}
$$

which exactly matches (19) for $\beta=1$.

We have proved

Proposition 7. The well-posed weak primal problem (23) leads directly to the well-posed weak dual problem (29).

Remark 6. The weak formulation (23) leads directly to the dual consistent formulation (29) for $\alpha=\beta=1$. It is straightforward to verify that $\alpha=$ $\beta=1$ satisfy conditions (18) and (22). Furthermore, they match the strong conditions (13) and (14).

\section{The discrete problem}

The derivation of the continuous primal and dual problems above provide a direct roadmap [14] for the numerical treatment in this section.

\subsection{The semi-discrete primal problem}

The semi-discrete finite difference scheme used in this paper is based on the SBP-SAT formulation [17, 18]. SBP operators are discrete differential operators which mimic the integration-by-part rule in the discrete setting. Consider a two-dimensional Cartesian equidistant grid of $\left(N_{x}+1\right)\left(N_{y}+1\right)$ points with coordinates $\left(x_{i}, y_{j}\right)$. In order to mimic the primal continuous weak formulation, the semi-discrete SBP-SAT approximation of (15) on the domain $(x, y) \in \Omega=[0,1] \times[0,1]$ is written as,

$$
\begin{aligned}
U_{t}+L_{h} U & =\tilde{F}, \quad t \geq 0 \\
U & =0, \quad t=0
\end{aligned}
$$

where $U=U(t)$ is the discrete solution, $\tilde{F}$ is the discrete form of $F$ and

$$
L_{h}=\left(D_{x} \otimes I_{y} \otimes A\right)+\left(I_{x} \otimes D_{y} \otimes B\right)-\left(P_{x}^{-1} E_{N_{x}} \otimes I_{y} \otimes \Sigma_{p} L_{p}\right) .
$$

The last term in (31) is the discrete lifting operator. For simplicity, we have only included the boundary conditions at $x=1$. The treatment of the remaining boundaries is similar. 
In (31), $D_{x, y}=P_{x, y}^{-1} Q_{x, y}$ is the difference operator, $P_{x, y}$ is a positive definite matrix and $Q_{x, y}$ satisfies $Q_{x, y}+Q_{x, y}^{T}=\operatorname{diag}[-1,0, \cdots, 0,1]$. The $x, y$ subscripts indicate differentiation in the corresponding coordinate direction. The projection matrix $E_{N_{x}}=\operatorname{diag}[0, \cdots, 0,1]$ is used to place the penalty term at the boundary point $x=1$. Moreover, $I_{x}$ and $I_{y}$ are the identity matrices of size $N_{x}+1$ and $N_{y}+1$. The boundary operator is $L_{p}$ and the penalty matrix $\Sigma_{p}$ is such that stability is achieved. Both $L_{p}$ and $\Sigma_{p}$ are already derived in the continuous setting, and given in (12) and (16), respectively.

\subsubsection{Stability}

The discrete energy method with $\tilde{F}=0$ (multiplying (30) from the left with $U^{T}\left(P_{x} \otimes P_{y} \otimes I_{m}\right)$ and adding the result to its transpose) leads to

$$
\begin{aligned}
\frac{d}{d t}\|U\|_{P_{x y}}^{2}= & -U^{T}\left(E_{N_{x}} \otimes P_{y} \otimes A\right) U+U^{T}\left(E_{N_{x}} \otimes P_{y} \otimes \Sigma_{p} L_{p}\right) U \\
& +\left(U^{T}\left(E_{N_{x}} \otimes P_{y} \otimes \Sigma_{p} L_{p}\right) U\right)^{T},
\end{aligned}
$$

where we have only kept the contribution from right boundary $(x=1)$. In (32), the semi-discrete norm is defined as $\|U\|_{P_{x y}}^{2}=U^{T} P_{x y} U$, where $P_{x y}=$ $\left(P_{x} \otimes P_{y} \otimes I_{m}\right)$ and $I_{m}$ is $m \times m$ identity matrix. The relation (32) can be simplified to

$$
\frac{d}{d t}\|U\|_{P_{x y}}^{2}=-U_{N_{x}}^{T}\left(P_{y} \otimes A\right) U_{N_{x}}+U_{N_{x}}^{T}\left(P_{y} \otimes \Sigma_{p} L_{p}\right) U_{N_{x}}+\left(U_{N_{x}}^{T}\left(P_{y} \otimes \Sigma_{p} L_{p}\right) U_{N_{x}}\right)^{T} .
$$

As in the continuous analysis, letting $\Sigma_{p}=X_{-}$and $L_{p}=\Lambda^{-} X_{-}^{T}-R X_{+}^{T}$, yield

$$
\frac{d}{d t}\|U\|_{P_{x y}}^{2}=-\left[\begin{array}{c}
W_{N_{x}}^{+} \\
W_{N_{x}}^{-}
\end{array}\right]^{T} P_{y} \otimes M(1)\left[\begin{array}{c}
W_{N_{x}}^{+} \\
W_{N_{x}}^{-}
\end{array}\right],
$$

where $M(1)(=\tilde{M}(1))$ is given in (17). Furthermore, the new variables are $W_{N_{x}}^{+}=\left(I_{y} \otimes X_{+}^{T}\right) U_{N_{x}}$ and,$W_{N_{x}}^{-}=\left(I_{y} \otimes X_{-}^{T}\right) U_{N_{x}}$. The matrix $M(1)$ is positive semi-definite and hence the following proposition holds.

Proposition 8. The primal semi-discrete approximation (30) with the weak boundary operator $L_{p}$ and penalty matrix $\Sigma_{p}=X_{-}$is a stable approximation of (1) and (15) if (13) holds.

Remark 7. The discrete derivation above amounts to administration and bookkeeping of the results already obtained in the continuous analysis. 


\subsection{A direct path to the semi-discrete dual problem}

In a similar manner as for the continuous problem in Section 3.3, we show how the discrete dual problem is obtained. To derive the semi-discrete dual problem, consider the linear functional $J_{h}(U, \tilde{G})=U_{h}^{T} P_{x y} \tilde{G}$, where $\tilde{G}$ is a vector weight function. As in the continuous case, we seek the discrete dual function $\Phi$ such that $\int_{0}^{T} J_{h}(U, \tilde{G}) d t=\int_{0}^{T} J_{h}(\Phi, \tilde{F}) d t$.

Integration by parts in time and inserting the initial and end conditions $u(x, y, 0)=\phi(x, y, T)=0$ gives

$$
\begin{aligned}
\int_{0}^{T} J_{h}(U, \tilde{G}) d t & =\int_{0}^{T} J_{h}(U, \tilde{G}) d t-\int_{0}^{T}\left(\Phi, U_{t}+L_{h}(U)-\tilde{F}\right)_{h} d t \\
& =\int_{0}^{T} J_{h}(\Phi, \tilde{F}) d t-\int_{0}^{T}\left(\Phi_{t}+\tilde{G}, U\right)_{h} d t-\int_{0}^{T}\left(\Phi, L_{h} U\right)_{h} d t .
\end{aligned}
$$

Again, we only consider the boundary at $x=1$ and ignore the terms related to the other boundaries. By using the SBP property $Q_{x}+Q_{x}^{T}=$ $\operatorname{diag}[-1,0, \cdots, 0,1]$, the last term in (34) can be rewritten as

$$
\begin{aligned}
& \left(\Phi, L_{h} U\right)_{h}=\Phi^{T} P_{x y} L_{h} U \\
= & +\Phi^{T}\left(\left(Q_{x} \otimes P_{y} \otimes A\right)+\left(P_{x} \otimes Q_{y} \otimes B\right)-\left(E_{N_{x}} \otimes P_{y} \otimes X_{-} L_{p}\right)\right) U \\
= & -\Phi^{T}\left(\left(Q_{x}^{T} \otimes P_{y} \otimes A\right)+\left(P_{x} \otimes Q_{y}^{T} \otimes B\right)+\left(E_{N_{x}} \otimes P_{y} \otimes X_{-} L_{p}-A\right)\right) U \\
= & -\Phi^{T}\left(\left(D_{x}^{T} \otimes I_{y} \otimes A\right)+\left(I_{x} \otimes D_{y}^{T} \otimes B\right)+\left(P_{x}^{-1} E_{N_{x}} \otimes I_{y} \otimes X_{-} L_{p}-A\right)\right) P_{x y} U \\
= & \left(L^{*} \Phi, U\right),
\end{aligned}
$$

where

$$
L_{h}^{*}=-\left(D_{x} \otimes I_{y} \otimes A^{T}\right)-\left(I_{x} \otimes D_{y} \otimes B^{T}\right)+\left(P_{x}^{-1} E_{N_{x}} \otimes I_{y} \otimes A^{T}-L_{p}^{T} X_{-}^{T}\right),
$$

with $L_{p}$ given in (12). At $x=1$, we have $C=A$ and the boundary operator in (36) can be simplified to

$$
A^{T}-L_{p}^{T} X_{-}^{T}=X_{+}\left(\Lambda^{+} X_{+}^{T}+R^{T} X_{-}^{T}\right)=X_{+} L_{d} .
$$

with $L_{d}$ given in (12). This yields

$$
L_{h}^{*}=-\left(D_{x} \otimes I_{y} \otimes A\right)-\left(I_{x} \otimes D_{y} \otimes B\right)+\left(P_{x}^{-1} E_{N_{x}} \otimes I_{y} \otimes X_{+} L_{d}\right),
$$


Substituting (35) into (34) and inserting the homogeneous initial conditions leads to

$$
\int_{0}^{T} J_{h}(U, \tilde{G}) d t=\int_{0}^{T} J_{h}(\Phi, \tilde{F}) d t+\int_{0}^{T}\left(\Phi_{t}-L_{h}^{*} \Phi+\tilde{G}, U\right)_{h} d t .
$$

The function $\Phi$ has to satisfy the discrete dual problem

$$
\begin{array}{rlrl}
\Phi_{\tau}+L_{h}^{*} \Phi & =\tilde{G}, & & \tau \geq 0 \\
\Phi & =0, & \tau=0
\end{array}
$$

when the time transformation $\tau=T-t$ is implemented. The discrete dual problem (37) is a consistent approximation of (5), including the dual boundary conditions (12), i.e. the scheme (30) is dual consistent.

We can also prove the following proposition.

Proposition 9. The semi-discrete dual approximation (37) with weak boundary operator $L_{d}$ and penalty matrix $\Sigma_{d}=-X_{+}$is a stable approximation of (5) and (19) if (14) holds.

Proof. The discrete energy method applied to the discrete dual problem (37) with $\tilde{G}=0$ results in

$$
\begin{aligned}
\frac{d}{d \tau}\|\Phi\|_{P_{x y}}^{2} & =\Phi^{T}\left(E_{N_{x}} \otimes P_{y} \otimes A\right) \Phi-\Phi^{T}\left(E_{N_{x}} \otimes P_{y} \otimes X_{+} L_{d}\right) \Phi \\
& -\left(\Phi^{T}\left(E_{N_{x}} \otimes P_{y} \otimes X_{+} L_{d}\right) \Phi\right)^{T}=\Phi_{N_{x}}^{T}\left(P_{y} \otimes A\right) \Phi_{N_{x}} \\
& -\Phi_{N_{x}}^{T}\left(P_{y} \otimes X_{+} L_{d}\right) \Phi_{N_{x}}-\left(\Phi_{N_{x}}^{T}\left(P_{y} \otimes X_{+} L_{d}\right) \Phi_{N_{x}}\right)^{T} \\
& =-\left[\begin{array}{l}
\Psi_{N_{x}}^{+} \\
\Psi_{N_{x}}^{-}
\end{array}\right]^{T} P_{y} \otimes \tilde{M}(1)\left[\begin{array}{l}
\Psi_{N_{x}}^{+} \\
\Psi_{N_{x}}^{-}
\end{array}\right],
\end{aligned}
$$

where $\tilde{M}(1)(=M(1))$ is given (21). Furthermore, the new variables are $\Psi_{N_{x}}^{+}=\left(I_{y} \otimes X_{+}^{T}\right) \Phi_{N_{x}}$ and $\Psi_{N_{x}}^{-}=\left(I_{y} \otimes X_{-}^{T}\right) \Phi_{N_{x}}$. The matrix $\tilde{M}(1)$ is positive semi-definite, which implies that the discretization (37) is stable.

Remark 8. Recall that for $\alpha=\beta=1$, the primal and dual equations have the same energy estimate in the continuous case (see (17) and (21)). Similar results are also obtained for the semi-discrete formulations of the primal problem in (33) and dual problem in (38). The similarities between the continuous and semi-discrete estimates are due to the use of SBP-SAT approximations. 
5 NUMERICAL EXPERIMENTS

\section{Numerical experiments}

To exemplify the theoretical results, consider the Maxwell's equations posed on the domain $\Omega=[0,1] \times[0,1]$,

$$
\begin{array}{rlrl}
\mu \frac{\partial H}{\partial t} & =-\nabla \times E, & \epsilon \frac{\partial E}{\partial t} & =\nabla \times H-J, \\
\nabla \cdot \epsilon E & =\rho, \quad \nabla \cdot \mu H=0 .
\end{array}
$$

Here, $E, H, \rho$ and $J$ represent the electric field-strength, the magnetic fieldstrength, the electric charge density, and the electric current density, respectively. Moreover, $\epsilon$ and $\mu$ are permittivity and permeability coefficients respectively [19].

With $J=0$ we can write $(39)$ in matrix form as

$$
S u_{t}+A u_{x}+B u_{y}=0
$$

where $u=\left[H_{z}, E_{x}, E_{y}\right]$ and

$$
S=\left[\begin{array}{lll}
\mu & 0 & 0 \\
0 & \epsilon & 0 \\
0 & 0 & \epsilon
\end{array}\right], \quad A=\left[\begin{array}{lll}
0 & 0 & 1 \\
0 & 0 & 0 \\
1 & 0 & 0
\end{array}\right], \quad B=\left[\begin{array}{ccc}
0 & -1 & 0 \\
-1 & 0 & 0 \\
0 & 0 & 0
\end{array}\right]
$$

In this example, we let $\rho=1, \mu=1$ and $\epsilon=1$. The matrices $A$ and $B$ are decomposed as $A=X_{A} \Lambda_{A} X_{A}^{T}$ and $B=X_{B} \Lambda_{B} X_{B}^{T}$, where

$\Lambda_{A}=\Lambda_{B}=\left[\begin{array}{ccc}1 & 0 & 0 \\ 0 & 0 & 0 \\ 0 & 0 & -1\end{array}\right], X_{A}=\left[\begin{array}{ccc}\frac{1}{\sqrt{2}} & 0 & \frac{1}{\sqrt{2}} \\ 0 & -1 & 0 \\ \frac{1}{\sqrt{2}} & 0 & \frac{-1}{\sqrt{2}}\end{array}\right], X_{B}=\left[\begin{array}{ccc}\frac{-1}{\sqrt{2}} & 0 & \frac{-1}{\sqrt{2}} \\ \frac{1}{\sqrt{2}} & 0 & \frac{-1}{\sqrt{2}} \\ 0 & 1 & 0\end{array}\right]$.

To test our method, we use the manufactured solution

$H_{z}=E_{z}=\sin (2 \pi x) \sin (2 \pi y)+3 \cos \left(\frac{\pi t}{2}\right), \quad E_{x}=\sin (2 \pi x) \sin (\pi y)+3 \cos \left(\frac{\pi t}{2}\right)$,

which provide all the data for the calculations. The boundary conditions are of the type (12) with $R_{E}=0, R_{S}=1, R_{W}=-1, R_{N}=0$, which denote the value of $R$ in (12) at the East, South, West, and North boundary, respectively (see Figure 1). 


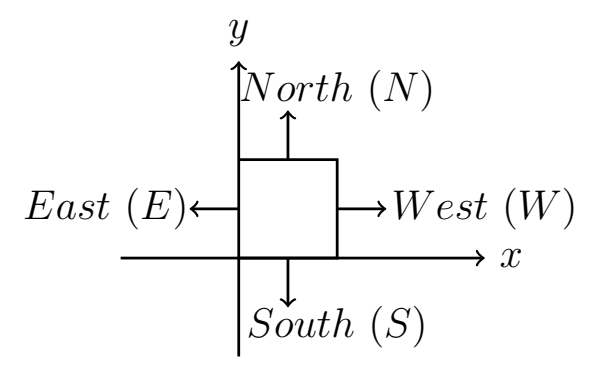

Figure 1: A schematic of the domain.

\subsection{Order of accuracy for the solution}

We examine the scheme for SBP operators of order $2 s$ in the interior and $s$ near the boundaries, where $s \in\{1,2,3,4\}$. The time integration is performed until time $T=1$ using the classical 4th-order Runge-Kutta method. The rates of convergence are calculated as $p=\log \left(E_{j} / E_{j+1}\right) / \log \left(N_{j+1} / N_{j}\right)$, where $N_{j}$ denotes the number of gridpoints at refinement level $j$ and $E_{j}$ is the error between the computed and exact solution for each variable. In Table 1, the convergence rates of the solutions are shown for a sequence of spatial mesh refinements. The results show that the design order of accuracy is obtained $[20,21]$.

\subsection{Order of accuracy for functionals}

In this subsection, we compute linear functionals to see if superconvergence is obtained $[9,10]$. We consider the linear functionals

$$
J\left(H_{z}\right)=\int_{\Omega} H_{z} d \Omega, \quad J\left(E_{x}\right)=\int_{\Omega} E_{x} d \Omega, \quad J\left(E_{y}\right)=\int_{\Omega} E_{y} d \Omega .
$$

By using the manufactured solution, the exact functionals can be computed.

Based on the theory derived earlier, the discrete scheme (30) is dual consistent for $\alpha=\beta=1$. If $\alpha \neq 1$ is chosen such that (18) is satisfied, then the scheme (30) is stable but not dual consistent. By choosing the penalty matrix at the north boundary as $\Sigma_{p}^{N}=2\left(X_{B}\right)_{-}$, a stable and dual inconsistence scheme is derived. The rates of convergence of the numerical functionals at $T=1$ for $\operatorname{SBP}(2,1), \operatorname{SBP}(4,2), \operatorname{SBP}(6,3)$ and $\operatorname{SBP}(8,4)$ for dual consistent and dual inconsistent scheme are given in Tables 2 and 3 , respectively. As shown in Table 2, superconvergence is achieved for the dual consistent discretization. 


\begin{tabular}{lllll}
\hline \hline$N=M$ & 20 & 40 & 80 & 120 \\
\hline $\mathrm{SBP}(2,1)$ & & & & \\
$H_{z}$ & 1.976 & 1.986 & 1.992 & 1.996 \\
$E_{x}$ & 2.013 & 2.004 & 2.001 & 2.000 \\
$E_{y}$ & 1.991 & 1.997 & 1.998 & 1.998 \\
& & & & \\
$\mathrm{SBP}(4,2)$ & & & & \\
$H_{z}$ & 3.028 & 3.053 & 3.029 & 3.017 \\
$E_{x}$ & 2.943 & 3.007 & 3.009 & 3.007 \\
$E_{y}$ & 2.983 & 3.031 & 3.016 & 3.009 \\
$\mathrm{SBP}(6,3)$ & & & & \\
$H_{z}$ & 4.783 & 4.795 & 4.619 & 4.441 \\
$E_{x}$ & 5.222 & 4.361 & 4.224 & 4.191 \\
$E_{y}$ & 5.395 & 4.588 & 4.350 & 4.332 \\
& & & & \\
$\mathrm{SBP}(8,4)$ & & & & \\
$H_{z}$ & 4.222 & 4.888 & 4.805 & 4.945 \\
$E_{x}$ & 5.655 & 5.552 & 5.442 & 5.400 \\
$E_{y}$ & 4.501 & 5.210 & 5.190 & 5.214 \\
\hline \hline
\end{tabular}

Table 1: Convergence rates for the solutions at $T=1$, for a sequence of mesh refinements. 


\begin{tabular}{lllll}
\hline \hline$N=M$ & 20 & 40 & 80 & 120 \\
\hline $\mathrm{SBP}(2,1)$ & & & & \\
$J\left(H_{z}\right)$ & 1.979 & 1.980 & 1.986 & 1.990 \\
$J\left(E_{x}\right)$ & 1.999 & 1.998 & 1.998 & 1.998 \\
$J\left(E_{y}\right)$ & 2.000 & 1.992 & 1.995 & 1.996 \\
& & & & \\
$\mathrm{SBP}(4,2)$ & & & & \\
$J\left(H_{z}\right)$ & 3.706 & 3.924 & 4.052 & 4.059 \\
$J\left(E_{x}\right)$ & 3.322 & 3.573 & 4.209 & 4.271 \\
$J\left(E_{y}\right)$ & 3.821 & 4.143 & 4.199 & 4.222 \\
$\mathrm{SBP}(6,3)$ & & & & \\
$J\left(H_{z}\right)$ & 5.881 & 6.013 & 5.902 & 5.899 \\
$J\left(E_{x}\right)$ & 5.842 & 6.081 & 6.069 & 5.995 \\
$J\left(E_{y}\right)$ & 5.915 & 6.103 & 6.103 & 6.108 \\
& & & & \\
$\operatorname{SBP}(8,4)$ & & & & \\
$J\left(H_{z}\right)$ & 4.821 & 7.552 & 7.932 & 8.032 \\
$J\left(E_{x}\right)$ & 5.332 & 7.697 & 7.899 & 7.902 \\
$J\left(E_{y}\right)$ & 4.814 & 7.777 & 8.012 & 7.999 \\
\hline \hline
\end{tabular}

Table 2: Convergence rates for the functionals at $T=1$, for a dual consistent scheme.

The boundedness of the error in the solutions and functionals for $\operatorname{SBP}(4,2)$ and $N=M=40$ grid points are shown in Figure 2. The results show that the error is bounded in time which should be the case according to the theory in [22]. Figure 3 shows the error of the solutions and functionals as a function of $\alpha$. It can clearly be seen that the errors are minimized for the dual consistent scheme, i.e. when $\alpha=1$.

\section{Summary and conclusions}

The relation between the primal and dual boundary conditions for linear hyperbolic systems of equations has been derived. By using this relation, well-posed dual/primal boundary conditions can be obtained from given wellposed primal/dual boundary conditions. A direct path from the weak primal 


\begin{tabular}{lllll}
\hline \hline$N=M$ & 20 & 40 & 80 & 120 \\
\hline $\mathrm{SBP}(2,1)$ & & & & \\
$J\left(H_{z}\right)$ & 1.990 & 1.987 & 1.990 & 1.993 \\
$J\left(E_{x}\right)$ & 2.003 & 2.0014 & 2.000 & 2.000 \\
$J\left(E_{y}\right)$ & 1.984 & 1.994 & 1.996 & 1.997 \\
& & & & \\
$\mathrm{SBP}(4,2)$ & & & & \\
$J\left(H_{z}\right)$ & 2.887 & 2.962 & 2.985 & 2.994 \\
$J\left(E_{x}\right)$ & 2.855 & 2.975 & 2.998 & 2.998 \\
$J\left(E_{y}\right)$ & 2.936 & 2.966 & 2.988 & 2.995 \\
& & & & \\
$\mathrm{SBP}(6,3)$ & & & & \\
$J\left(H_{z}\right)$ & 5.464 & 5.460 & 4.931 & 4.448 \\
$J\left(E_{x}\right)$ & 5.517 & 5.541 & 5.044 & 4.532 \\
$J\left(E_{y}\right)$ & 5.237 & 5.296 & 4.973 & 4.572 \\
& & & & \\
$\mathrm{SBP}(8,4)$ & & & & \\
$J\left(H_{z}\right)$ & 5.222 & 5.321 & 5.298 & 5.382 \\
$J\left(E_{x}\right)$ & 4.442 & 4.825 & 4.802 & 4.881 \\
$J\left(E_{y}\right)$ & 3.242 & 5.012 & 4.922 & 4.906 \\
\hline \hline
\end{tabular}

Table 3: Convergence rates for the functionals at $T=1$, for a dual inconsistent scheme.
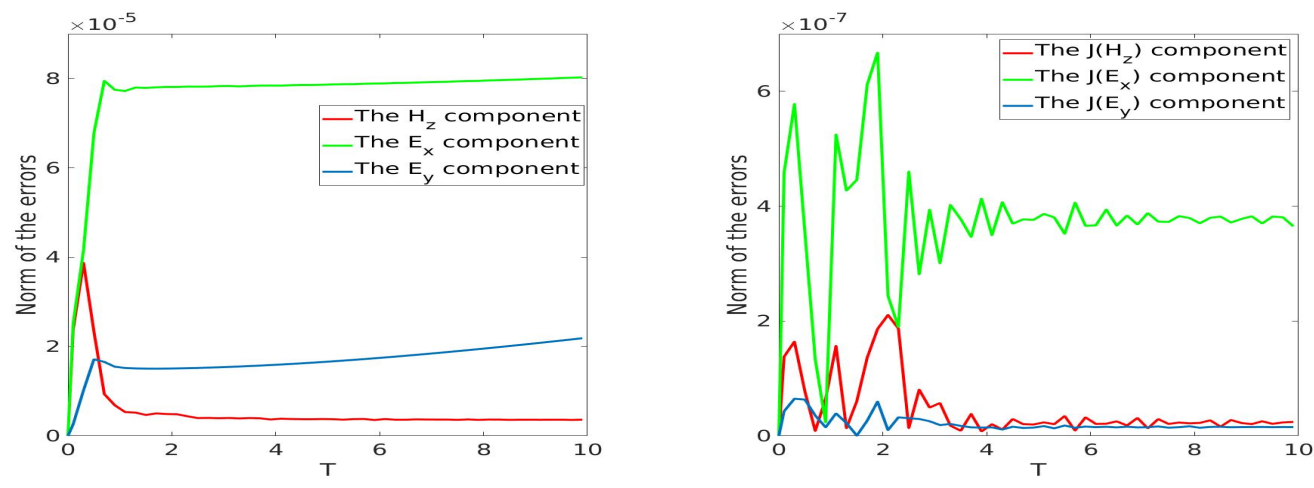

Figure 2: Error in the solutions and functionals as a function of time using $N=M=40$ grid points and $\operatorname{SBP}(4,2)$ for dual consistent case. 

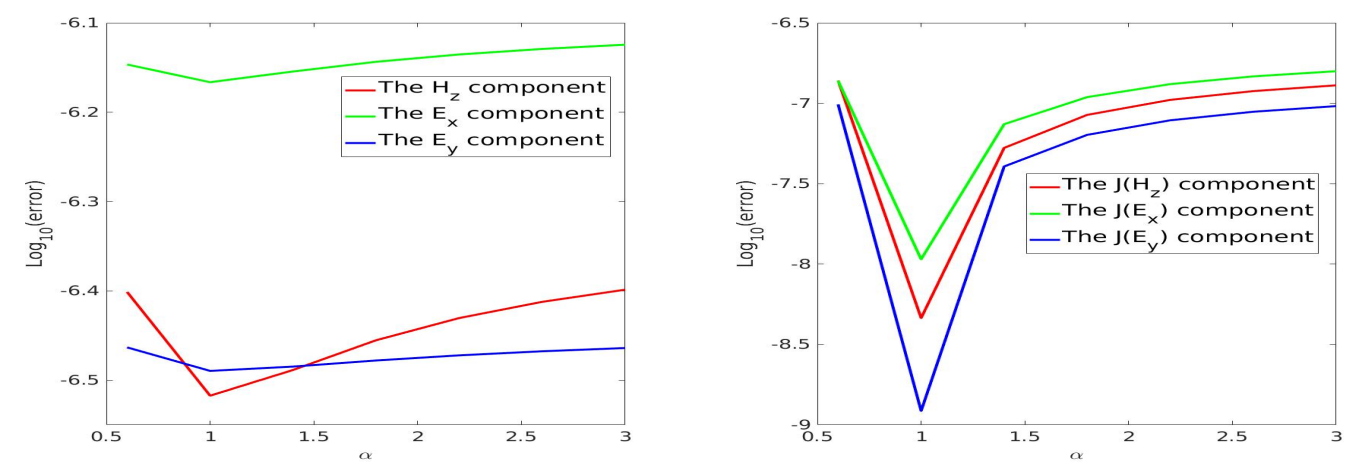

Figure 3: Error in the solutions (left) and functionals (right) as a function of $\alpha$ using $N=M=40$ grid points and $\operatorname{SBP}(4,2)$.

problem to the weak dual problem has also been presented and penalty coefficients are determined such that a dual consistent formulation is achieved.

It has also been shown that the weak boundary procedures in the wellposedness analysis lead directly to stability of the numerical approximation on SBP-SAT form. Superconvergence of the functionals was illustrated using the two-dimensional Maxwell's equations. Finally, it was illustrated that some penalty coefficients lead to stability but dual inconsistency, where the superconvergence is destroyed.

\section{References}

[1] N. A. Pierce, M. B. Giles, Adjoint recovery of superconvergent functionals from pde approximations, SIAM Review 42 (2000) 247-264.

[2] N. A. Pierce, M. B. Giles, Adjoint and defect error bounding and correction for functional estimates, Journal of Computational Physics 200 (2004) 769-794.

[3] M. B. Giles, N. A. Pierce, On the properties of solutions of the adjoint Euler equations, Numerical Methods for Fluid Dynamics VI ICFD (1998) 1-16.

[4] M. Paraschivoiu, J. Peraire, A. Patera, A posteriori finite element bounds for linear- functional outputs of elliptic partial differential equa- 
tions, Computer Methods in Applied Mechanics and Engineering 150 (1997) 289-312.

[5] P. Monk, E. Süli, The adaptive computation of far-field patterns by a posteriori error estimation of linear functionals, SIAM Journal on Numerical Analysis 36 (1998) 251-274.

[6] Y. Cheng, C. W. Shu, Superconvergence of discontinuous Galerkin and local discontinuous Galerkin schemes for linear hyperbolic and convection-diffusion equations in one space dimension, SIAM Journal on Numerical Analysis 47 (2010) 4044-4072.

[7] A. Ern, J. Proft, A posteriori discontinuous Galerkin error estimates for transient convection-diffusion equations, Applied Mathematics Letters 18 (2005) 833-841.

[8] T. Lundquist, J. Nordström, The SBP-SAT technique for initial value problems, Journal of Computational Physics 270 (2014) 86-104.

[9] J. E. Hicken, D. W. Zingg, Superconvergent functional estimates from summation-by-parts finite-difference discretizations, SIAM Journal on Scientific Computing 33 (2011) 893-922.

[10] J. Berg, J. Nordström, Superconvergent functional output for timedependent problems using finite differences on summation-by-parts form, Journal of Computational Physics 231 (2012) 6846-6860.

[11] J. Berg, J. Nordström, On the impact of boundary conditions on dual consistent finite difference discretizations, Journal of Computational Physics 236 (2013) 41-55.

[12] J. Berg, J. Nordström, Duality based boundary conditions and dual consistent finite difference discretizations of the Navier-Stokes and Euler equations, Journal of Computational Physics 259 (2014) 135-153.

[13] S. Abarbanel, D. Gottlieb, Optimal time splitting for two- and threedimensional Navier-Stokes equations with mixed derivatives, Journal of Computational Physics 41 (1981) 1-43.

[14] J. Nordström, A roadmap to well posed and stable problems in computational physics, Journal of Scientific Computing 71 (2017) 365-385. 
[15] F. Ghasemi, J. Nordström, Coupling requirements for multi-physics problems posed on two domains, SIAM Journal on Numerical Analysis 55 (2017) 2885-2904.

[16] D. N. Arnold, F. Brezzi, B. Cockburm, L. D. Marini, Unified analysis of discontinuous Galerkin methods for elliptic problems, SIAM journal on numerical analysis 39 (2002) 1749-1779.

[17] M. Svärd, J. Nordström, Review of summation-by-parts schemes for initial-boundary-value problems, Journal of Computational Physics 268 (2014) 17-38.

[18] D. C. Del Rey Fernández, J. Hicken, D. Zingg, Review of summationby-parts operators with simultaneous approximation terms for the numerical solution of partial differential equations, Computers \& Fluids 95 (2014) 171-196.

[19] J. Nordström, R. Gustafsson, High order finite difference approximations of electromagnetic wave propagation close to material discontinuities, Journal of Scientific Computing 18 (2003) 215-234.

[20] B. Strand, Summation by parts for finite difference approximations for d/dx, Journal of Computational Physics 110 (1994) 47-67.

[21] M. Svärd, J. Nordström, On the order of accuracy for difference approximations of initial-boundary value problems, Journal of Computational Physics 218 (2006) 333-352.

[22] J. Nordström, Error bounded schemes for time-dependent hyperbolic problems, SIAM Journal on Scientific Computing 30 (2007) 46-59. 